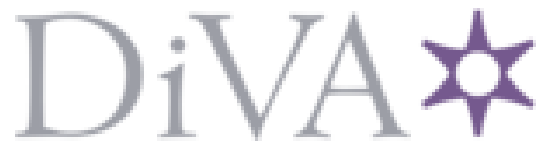

http://www.diva-portal.org

Preprint

This is the submitted version of a paper published in Journal of Economic Psychology.

Citation for the original published paper (version of record):

Farjam, M. (2019)

On whom would I want to depend; humans or computers?

Journal of Economic Psychology, 72: 219-228

https://doi.org/10.1016/j.joep.2019.04.002

Access to the published version may require subscription.

N.B. When citing this work, cite the original published paper.

Permanent link to this version:

http://urn.kb.se/resolve?urn=urn:nbn:se:lnu:diva-81815 


\title{
On whom would I want to depend; Humans or Computers?
}

\author{
Mike Farjam ${ }^{\mathrm{a}, \mathrm{b}}$ \\ ${ }^{a}$ Department of Social Studies, Linnaeus University, Växjö, Sweden \\ ${ }^{b}$ Linnaeus University Centre for Data Intensive Sciences \& Applications (DISA@LNU), Växjö, \\ Sweden
}

\begin{abstract}
We study in a laboratory experiment whether humans prefer to depend on decisions of others (Human-Driven Uncertainty) or states generated by a computer (Computerized Uncertainty). The experimental design introduced in this paper is unique in that it introduces Human-Driven Uncertainty such that it does not derive from a strategic context. In our experiment, Human-Driven Uncertainty derives from decisions, which were taken in a morally neutral context and in ignorance of externalities that the decisions may have on others. Our results indicate that even without strategic interaction and moral elements humans prefer Computerized to Human-Driven Uncertainty. This holds even when the distribution of outcomes under both types of uncertainty is identical. From a methodological point of view, the findings shed a critical light on behavioral research in which it is common practice to control for strategic uncertainty by comparing interaction with an artificial agent with a known strategy to interaction with humans. Outside the laboratory, our results suggest that whenever dependence on humans is changed to dependence on computers and other kinds of "artificial" decision makers, preferences with regard to these dependencies may change too.
\end{abstract}

Keywords: Ambiguity aversion, Experiment, Risk, Human uncertainty

\footnotetext{
*Correspondance: Department of Social Studies, Linnaeus University, 35195 Växjö, Sweden Email address: mike.farjam@lnu.se (Mike Farjam)
} 


\section{Introduction}

The laboratory experiment presented in this paper studies whether humans prefer to depend on states generated by a computer (Computerized Uncertainty) or other humans' decisions (Human-Driven Uncertainty). In our lottery choice experiment, subjects can choose the exact type of uncertainty on which they depend. Different from previous experiments studying these types of uncertainty, the Human-Driven Uncertainty in our experiment does not derive from choices of others taken in a game or any other kind of strategic context, and the decisions that other humans took were not morally loaded (contrary to e.g., choosing the strategy in a Trust Game). This operationalization of Human-Driven Uncertainty allows us a clean comparison of preferences with regard to Human-Driven versus Computerized Uncertainty.

Of course, rational, expected value maximizing agents should not care about the source of uncertainty. Humans however, being far from perfectly rational, may (e)valuate both types of situations very differently in terms of subjective probabilities and emotions involved. As evidence of this, Abdellaoui et al. (2011) find that subjects prefer to depend on lotteries where the uncertainty of winning is determined by the weather (also referred to as Natural Uncertainty), compared to a stock market and McCabe et al. (2003) find that humans also care about the intentions on which a decision was based.

A clean comparison between Computerized and Human-Driven Uncertainty is hard to achieve in the laboratory. Human-Driven Uncertainty arises naturally in what is referred to as strategic interaction by economists, defined as two or more human decision makers depending on each others choices (Van Huyck et al. 1991). This is why (to the best of our knowledge) in all experiments studying 
Human-Driven Uncertainty, the uncertainty derives from someone's action in a game-like setting. Section 2.1 reviews these experiments and section 2.2 discusses potential neural and psychological mechanisms leading to different behavior under both kinds of uncertainty.

Carrubba et al. (2007) argue that strategic interaction ${ }^{1}-$ to some extent - can be seen as a lottery, where one depends on the actions of others without knowing which action others will take. Strategic uncertainty, however, differs in more than one aspect from non-human based uncertainty. While non-human sources of uncertainty involve only oneself depending on a mechanism without intentions, strategic uncertainty includes interdependence between humans, and as a consequence a decision maker forms beliefs about others intentions. Furthermore, the options that one can choose from create (often morally loaded) externalities on others, which may lead to social preferences over outcomes. Dana et al. (2007) show that when subjects have to take decisions in a morally loaded context they actually prefer uncertainty on how their decision affects others. Summarized, one is not making a ceteris paribus comparison when comparing non-human sources of uncertainty and strategic uncertainty. To overcome this flaw, we designed an experiment in which the only difference between a the different types of uncertainty is the source of uncertainty. The Human-Driven Uncertainty involved in treatments of the experiment will therefore not derive from strategic interaction. We encounter Human-Driven Uncertainty that does not arise from strategic interaction regularly. Examples are situations where we depend on decisions that others took without knowing the consequences. Often, we even depend on actions of others that are not the result of any conscious decision at all. As an example, an

\footnotetext{
${ }^{1}$ Note, the term strategic as used by economists refers to to decision makers depending on each other and is different from the meaning of the word in everyday language.
} 
employee opening an email attachment that contains a computer virus may be absolutely unaware of causing harm to the employer. For the employer, the source of uncertainty is not the product of a strategic decision of the employee, although the source of uncertainty is the employee's behavior. In addition to the differentiation between Computerized and Human-Driven Uncertainty we also control the measurability of uncertainty (Knight 1921). "Measurable" uncertainty (henceforth: risk) is characterized by a situation in which the probabilities with which all possible events occur are known. "Unmeasurable" uncertainty (henceforth: ambiguity ${ }^{2}$ ) is characterized by the absence of known probabilities. In his seminal thought experiment, Ellsberg (1961) argues that humans generally avoid ambiguous lotteries in favor of lotteries were the distribution is known. Many experiments have since found that humans usually are ambiguity averse (Camerer and Weber 1992).

Table 1 shows the 4 possible combinations of the two dimensions along which uncertainty will be distinguished in this paper. The treatments in the experiment (presented in section 3) correspond to the cells in the table.

The research questions of this paper are:

1. Do humans prefer lotteries with Human-Driven Uncertainty to Computerized Uncertainty or vice versa?

2. Do humans have different risk preferences in lotteries where they depend on humans instead of computers?

3. Do we see ambiguity preferences under Human-Driven Uncertainty?

\footnotetext{
${ }^{2}$ In some parts of the literature this is referred to as uncertainty. Unfortunately there is no consistency with using these terms in the literature.
} 


\begin{tabular}{|c|c|c|}
\hline & Distribution known & Distribution unknown \\
\hline Human & Human-Driven Risk (HR) & Human-Driven Ambiguity (HA) \\
\hline Computer & Computerized Risk (CR) & Computerized Ambiguity (CA) \\
\hline
\end{tabular}

Table 1: Uncertainty concepts implemented

\section{Literature}

\subsection{Experiments on different sources of uncertainty}

Heinemann et al. (2009) elicit certainty equivalents of two lotteries in an experiment. In the first lottery the probability distribution of winning and losing depends on the actions of other players in a coordination-game, in the second lottery the outcome depends on the role of a die. They find that certainty equivalents of both lotteries were similar. From a ceteris paribus point of view the comparison Heinemann et al. are making is problematic. Additional to the source of uncertainty their treatments differ, since the roll of a die is characterized by known probabilities and thus a decision problem under risk, while the strategic uncertainty condition is characterized by ambiguity. Furthermore, all arguments made earlier when it comes to comparing a game with a decision problem apply. Li et al. (2018) show that ambiguity preferences indeed play a role in deciding whether or not to trust a trustee in a Trust Game. Although controlling for beliefs about the trustworthiness of the other, they do not explore whether these preferences also depend on other (strategically not relevant) properties that the other human may have.

Bohnet et al. (2008) addressed many of the issues when it comes to a clean comparison between risk and strategic/Human-Driven Uncertainty. In their experiment subjects play a binary Trust Game, where half of the subjects are first-movers (Trustors) and the other half second-movers (Trustees). They ask 
Trustors for the minimum acceptable probability (MAP) of playing against a trustworthy Trustee for which they would be willing to make a trust move. Trustors and Trustees are then randomly matched and Trustors will play the trust move if their MAP is smaller than the fraction of trustworthy Trustees. In asking for the MAP Bohnet et al. transform the strategic interaction to a risky decision problem. They find that MAPs of Trustors are generally higher when the Trustee's move is based on a decision compared to when the strategy was determined by a chance mechanism. The fact that Trustors asked for an extra risk premium when depending on humans compared to depending on a mechanism is interpreted as evidence for "betrayal aversion". We argue that Bohnet et al. jump to conclusions since as in Heinemann et al. (2009) they compare a situation where one depends on a neutral mechanism without any intentions with a situation where one depends on a human who had (morally loaded) intentions when taking the conscious decision. An alternative explanation for the finding of Bohnet et al. may be what one could refer to as "human aversion" (humans just do not like to depend on others).

Similar to Bohnet et al. (2008), Fairley et al. (2016) let subjects play a Trust Game as both Trustee and Trustor. When in the role of a Trustor, subjects were assigned to four random Trustees and one random decision of the Trustees was played. There were thus five potential probabilities of encountering a trustworthy Trustee depending on the number of trustworthy Trustees. Trustors had to indicate the amount that they would be willing to pay in order to play the lottery for each potential probability. Fairley et al. compare the amounts per probability with amounts that had to be indicated for standard risky lotteries with the same 5 potential probabilities of winning. They find that choices in lotteries and social lotteries are not correlated.

The experiments discussed do not provide a clear picture of how the various 
operationalizations of strategic and Human-Driven Uncertainty on the one hand and Natural and Computerized Uncertainty on the other relate to each other. However, since all results were obtained by comparing non-human based uncertainty with uncertainty based on some strategic action of another human, it remains unclear how these results relate to a comparison without a strategic component.

\subsection{Potential psychological and neural mechanisms}

Experiments in the area of neuroeconomics indicate that subjects react differently to situations when humans are involved. In Human-Driven Uncertainty, adding other humans as a part of the decision making process may automatically activate brain areas, emotions, and behavioral scripts that are usually used in social context.

Lauharatanahirun et al. (2012) study differences in brain activity (through fMRI) when subjects engage in a Trust Game compared to a lottery where the outcome depends on a random mechanism. They find that especially the amygdala (related to emotions) is more active in the Trust Game. Sanfey (2007) argues that emotions are crucial in economic decisions that involve other humans and brain areas that involve emotion regulation are therefore more active in situations with Human-Driven Uncertainty. Sanfey et al. (2003) find that the anterior insula is more active when subjects get unfair offers in an Ultimatum Game where the proposer is a human compared to the proposer being a chance mechanism. The latter seems to evoke fewer emotions than interaction with humans. This finding is robust across different types of games like Rock-Paper-Scissors (Chaminade et al. 2012), prisoners dilemma game (Krach et al. 2008; Rilling et al. 2004) and Trust Games (McCabe et al. 2001). These experiments also show that humans invest more effort when their counterpart is human. 
McCabe et al. (2001) and Rilling et al. (2004) similarly find that brain areas known to be crucial for theory of mind (the ability to put oneself into the shoes of someone else) are more active when subjects know they are playing with humans than when they know they are playing with computers. On the same line Rilling et al. (2002) study activation in the striatum, which is a crucial brain area when social decisions have to be made, when subjects interact with computers and robots. This area is less active when dealing with situations that involve Computerized Uncertainty.

Although the neuroeconomic literature shows that from a perceptional point uncertainty is treated differently when humans are the source compared to non-human sources, the literature does not point to a clear prediction on whether humans would prefer either of the types of uncertainty.

\section{Methods}

\subsection{General design}

Each subject was confronted with four lotteries in a random order. Each of these lotteries contained one of the kinds of uncertainty presented in Table 1. After each lottery the subjects indicated (through a multiple price list) how high a safe payoff would need to be in order for them to not engage in the lottery. After the four lotteries, subjects had to play one of the previous lotteries an additional time and could indicate which of those they would prefer. The entire experiment was computerized using z-Tree (Fischbacher 2007).

\subsection{Treatments}

In the lotteries in treatment CR (Computerized Risk) and CA (Computerized Ambiguity) subjects had to guess the result of a virtual card draw. Cards were drawn from a virtual stack of 10 cards. Each card was either an $A$ - or a $B$-card. In 
CR the distribution was 50/50 and subjects were explicitly told so. In CA subjects did not know the distribution and were told that any number of $A$-cards from 0-10 was possible and that the remaining of the 10 cards were B-cards. The computer would randomly select one of the virtual cards and subjects had to guess if that card was an $A$ - or a $B$-card. If their guess was correct, they would receive 100 ECU for that task otherwise 0. 10 ECU were worth 1 Euro. The lotteries in treatments HR (Human-Driven Risk) and HA (Human-Driven Ambiguity) were almost identical to those in CR and CA. Before the experiment, a survey was conducted among students that did not participate in the experiment and this survey was then used in the experiment to generate Human-Driven Uncertainty. In treatments HR and HA instead of cards, the computer would draw a random answer of a participant from the survey. Subjects in the experiment were told that 10 participants of the survey had to indicate their preference towards two different pictures. Subjects got no information on the content of pictures or the artists. They were only told that the one picture would be referred to as picture $A$ and the other as $B$. The task of the subject was to guess whether the participant of the survey drawn by the computer preferred picture $A$ or $B$. In HR subjects knew that the distribution was 50/50 and in HA the distribution was unknown to the subject. It was also made clear that the participants in HR are not the same participants as in HA. ${ }^{3}$

\subsection{Dependent variables}

We followed the recommendation of Trautmann et al. (2011) to use Certainty Equivalence and a direct comparison of different types of uncertainty to measure

\footnotetext{
${ }^{3}$ This surveys, of course, really took place, done with pictures from Klee and Kandinsky on the campus of the university in Jena.
} 
ambiguity aversion. After subjects played all the lotteries in all 4 treatments they were presented with a table (Appendix A.5) in which the rows contained all possible pairwise comparisons between the 4 treatments. Per pair of treatments subjects indicated which of the treatments they would prefer to play as an additional 5th treatment. One of these pairs of treatments was chosen randomly by the computer and the subject's preferred treatment was then played. To rule out order effects with regard to the order in which treatments are paired, the comparison that subjects had to make within each pair was not directly about treatments, but about rounds. Since the treatment per round was determined randomly, the treatments in each row of the comparison table also have a random order per subject.

As a second elicitation mechanism subjects had to fill in a multiple price list (Appendix C) in every treatment after taking their guess on $A$ or $B$ in the lottery. In this multiple price list subjects had to choose 10 times between a secure payoff that ranges from 9 to $99 \mathrm{ECU}$ in steps of $10 \mathrm{ECU}$ and an uncertain payoff which would be either 0 or 100 ECU depending on the result of the lottery. The relative frequency of lottery-choices was used as a measure for preference for that lottery.

\subsection{Instructions and controls}

Before subjects got instructions per treatment they watched a video (2:36 minutes) with general information. ${ }^{4}$ Appendix A contains the full set of instructions describing the lotteries in each treatment.

To avoid differences in preferences regarding the treatments being potentially driven by complexity aversion (Sonsino et al. 2002), the instructions per treatment were as similar as possible in terms of length, wording and syntactic structure.

\footnotetext{
${ }^{4}$ Video at https://osf.io/pa8zm/?view_only=4664944291954848a128220a764b3057
} 


\begin{tabular}{|c|c|c|c|}
\hline Treatment & Sentences & Words & Characters \\
\hline \hline Computerized Risk & 4 & 51 & 272 \\
\hline Computerized Ambiguity & 4 & 54 & 288 \\
\hline Human-Driven Risk & 4 & 47 & 288 \\
\hline Human-Driven Ambiguity & 4 & 51 & 301 \\
\hline
\end{tabular}

Table 2: Comparing the complexity of the (German) instructions in each treatment

Table 2 gives a rough overview of the complexity of the instructions in each treatment.

To avoid order effects the treatments/lotteries were presented in a random order per subject. To control whether subjects understood the distribution of cards in a treatment, they were asked at the end of the experiment how many $A$ - and $B$-cards/survey answers were in each of the stacks/surveys per treatment. The answers were incentivized with 2 ECU for correctness. We also asked subjects after the experiment the question "Do you trust that the experimentors in this lab truthfully conducted the procedures as presented to you in the instructions?" in order to identify subjects that may believe that random draws or the survey were rigged. None of the subjects answered "No" to the question.

\section{Results}

\subsection{Subjects}

Eighty-eight subjects participated in this study. Since studies like Dohmen et al. (2011) show that risk-preferences differ with gender, only male subjects were recruited to reduce within sample variability. All sessions were run in May 2015 in the laboratory of the Friedrich Schiller University Jena. Seventy-six\% of our subjects were students. 


\begin{tabular}{rrrr}
\hline & CR & CA & HR \\
\hline CA & 0.0000 & & \\
HR & 0.0018 & 0.0000 \\
HA & 0.0000 & 0.5059 & 0.0000 \\
p-values adjusted for multiple testing with the Holm-Bonferroni method.
\end{tabular}

Table 3: P-values of a pairwise comparison between treatments with a Wilcox rank-sum test

\subsection{Payoff}

To avoid endowment effects only one of the treatments was chosen randomly at the end of the session for payoff. Subjects earned on average 7 Euro (including a 2.5 Euro show-up fee). The experiment (including the payment) took about 30 minutes.

\subsection{Pairwise comparison}

This subsection compares how often subjects preferred one treatment over one of the other 3 treatments. Thus, the maximum number of times a lottery could be preferred above other lotteries is 3 and the minimum is 0 . Table 3 shows the result of a pairwise comparison of all 4 treatments.

From Table 3 and Figure 1 (left) we can derive the following order of preferences: $C R \succ H R \succ C A \sim H A$. The fact that $C R \succ C A$ and $H R \succ H A$ indicates that subjects were ambiguity averse. Ambiguity aversion thus seems to be found not just in nature uncertainty but also under Human-Driven Uncertainty. Most importantly, we find that subjects prefer CR to HR and $66 \%$ of subjects preferred CR to HR in the direct comparison.

To check whether the results are driven by an order effect (despite the randomization of the order of treatments per subject) two mixed effects models were used to predict the preferences of subjects regarding a treatment. An 

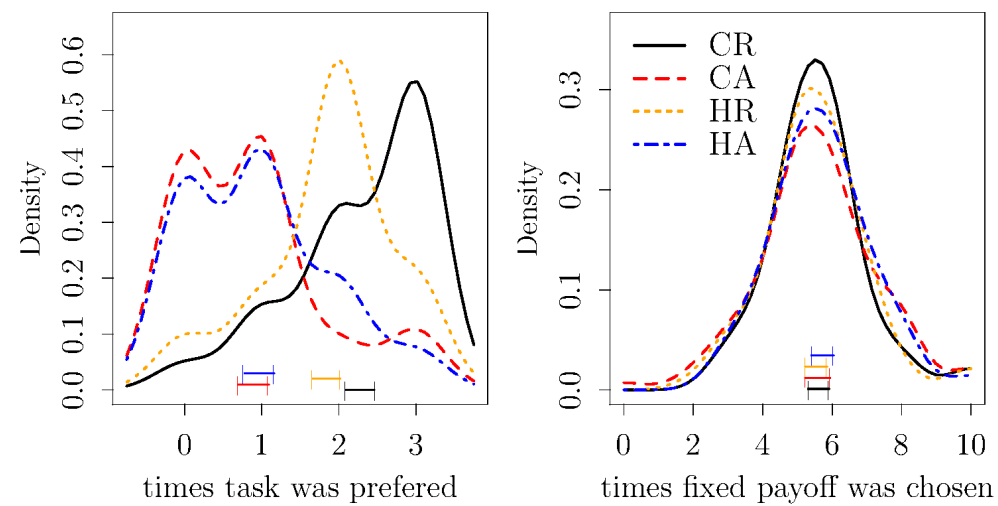

Per distribution of answers the $95 \%$ bootstrap confidence interval of the mean is shown.

Figure 1: Left, distributions on how often subjects preferred each treatment above the other 3 treatments. Right, distributions of choices for the lottery instead of the safe payment per treatment in the multiple price list.

ANOVA comparing the model with and without the order of treatments as a fixed factor shows that the model without order performs better in terms of AIC. The preferences subjects had regarding the treatments thus do not seem to be affected by the order in which the treatments were presented.

\subsection{Multiple price list}

Subjects had to choose 10 times between a fixed secure payoff and the uncertain payoff of a lottery. The expected payoff of all lotteries was 50 ECU. Hence rational, risk-neutral agents would choose the payoff of the lottery if the fixed payoff is below $50 \mathrm{ECU}$ and choose the fixed payoff if it is above $50 \mathrm{ECU}$. Given the multiple price list subjects were confronted with (see Appendix C) one would expect that subjects choose 5 times the fixed payoff and 5 times the payoff of the treatment.

On average subjects chose the fixed payoff 5.6 times, which is more often than a 
rational, risk-neutral agent would and which indicates that subjects were (as usually found) risk-averse. Thirty-three\% of subjects chose the fixed lottery equally often in all treatments, indicating that they were indifferent between all treatments. Figure 1 (right) shows that the distributions of choices for the payoff of the lottery per treatment are almost identical. Furthermore, $12.5 \%$ of subjects had more than one switching point in the multiple price list and $10 \%$ of subjects had more than 1 switching point which indicates that some did not understand how a multiple price list works. However, multiple switching points are often found when using multiple price lists for preference elicitation (Andersen et al. 2006). ${ }^{5}$

Summarized, analysis of the data with regard to choices in the multiple price list seems problematic. The statistical analysis of subjects' choices in the multiple price list will therefore only be rudimentary.

A Friedman-test comparing the mean number of times the lottery was preferred above the fixed amount shows that the differences between treatments with regard to choices in the multiple price list were not significant $\left(\mathrm{p}=0.37, \chi^{2}=3.16\right)$. A Bartlett-test comparing the variance between groups finds that the only significant difference regarding variance can be found between treatments CR and CA (not adjusted for multiple testing: $\left.\mathrm{p}=0.029, K^{2}=4.77\right)$. Neither of the other comparisons lead to p-values below the 0.1 significance level. Looking at Figure 1 (right) it seems unlikely that differences would become significant with a larger sample.

It thus seems that although the previous subsection shows that subjects prefer

\footnotetext{
${ }^{5}$ Appendix B shows the results of a pilot study of this experiment where a BDM mechanism (Becker et al. 1964) was used to elicit preferences. Results obtained by the BDM mechanism are almost the same as with the multiple price list.
} 
some treatments above others, the treatment they are in does not change their risk-preference regarding an opt-out option from that treatment. This is in line with other research, often under the label of the comparative ignorance hypothesis, finding that preferences with regard to different types of uncertainties can be best identified when directly contrasting the types of uncertainty (Fox and Tversky 1995; Chow and Sarin 2001; Trautmann et al. 2011).

\subsection{Robustness checks}

To check whether the results may be driven by different beliefs about the distributions in each treatment subjects had to guess the number of $A$ 's in each treatment. Figure 2 (left) shows that on average subjects had the same expectations in all treatments and that the great majority in CR and HR understood that there were exactly $5 A$ 's in these treatments.

Furthermore, we measured per subject how long they remained on screens that presented the description of a treatment (the exact instructions can be found in Appendix A). The time spent on this screen can be seen as a proxy for the perceived complexity of the description. Figure 2 (right) shows that there was hardly any difference between treatments CA and HA and treatments CR and HR. However, subjects remained on average 7.2 seconds longer on the screens that presented instructions in treatment CA than in treatment CR and 6.2 seconds longer on screens of HA than HR.

In summary, the fact that subjects preferred CR to HR does not seem to be driven by a misunderstanding of the two treatments or differences in perceived complexity. However, the fact that subjects generally prefer HR to HA and CR to CA may be explained by both: ambiguity and complexity aversion. 

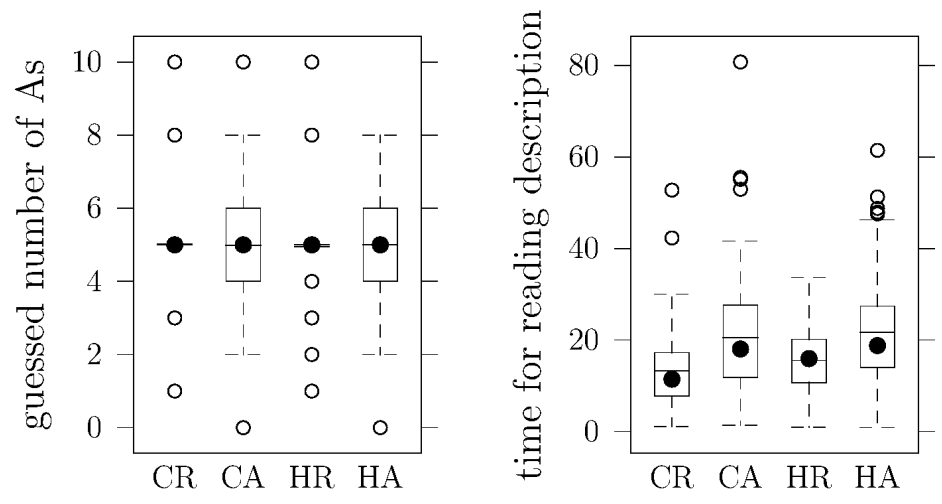

Box-and-whisker plots per treatment. Dots represent medians, lines means.

Figure 2: Left, subjects' believe about the frequency of $A$ 's. Right, time spend for reading description on distribution of $A$ 's

\section{Discussion}

In the experiment presented, subjects had the choice between a lottery where they depend on a distribution of outcomes based on decisions of humans and a lottery where the distribution does not depend on humans but only on the computer.

When the distribution of outcomes was ambiguous, preferences with regard to both lotteries were on average the same. In the risky lotteries where the distribution of outcomes was known and identical, subjects clearly preferred to depend not on other humans but on the computer.

Bohnet et al. show that humans demand a "risk premium" in order to accept strategic dependence on another human compared to a chance mechanism. We show that humans demand this premium even without any strategic interdependence. While Bohnet et al. conclude that subjects are betrayal averse, our results suggest that humans simply prefer to not depend on others, irrespective of any betrayal. 
To the best of our knowledge this experiment shows for the first time that humans care about the fact that they depend on other humans irrespective of the outcome and even in the absence of intentions of the human on whom they depend. This suggests that differences in perception between Human-Driven and Computerized Uncertainty are more fundamental than expected. McCabe et al. (2003) claim that humans do not just care about the probabilities with which an event occurs but also about the process that led to these probabilities. In our experiment, we find that even when the distribution on which an outcome in a lottery depends is identical and known, humans may systematically prefer one lottery to the other. In our experiment subjects had clearly different preferences regarding Computerized and Human-Driven Risk, yet they did not have different risk preferences once facing one of the two types of risk in a lottery. This may mean that humans, given the choice whether they want to depend on humans or computers, would choose computers, but that once they are in a specific situation do not differ in terms of behavior in both conditions. However, risk preferences are not the only indicator for behavior and future studies are needed to look more carefully at how human behavior differs under Computerized and Human-Driven Risk.

We can only speculate on the reasons why subjects preferred to depend on computers and not on other humans' decisions. Besides the differences in the neural mechanism that may work depending on the kind of risk (see section 2.2), Heath and Tversky (1991) show that humans generally prefer to engage in lotteries which they know more about or feel more knowledgeable. This holds even if the knowledge is not relevant for the outcome of the lottery (Maffioletti and Santoni 2005). Subjects in the experiment may have felt less knowledgeable about lotteries with Human-Driven Uncertainty compared to Computerized Uncertainty, simply because being confronted with decisions of others was 
perceived as less transparent by subjects than being confronted with the letter on a card.

The results we obtained also have important methodological implications. An often used method to control for strategic uncertainty in an economic game is to have a baseline condition with the normal game and a treatment condition with a computerized player where the algorithm of the computer is known to subjects (e.g., Koch and Penczynski 2018). Our results however show that playing against a computer may be perceived as fundamentally different from playing with and depending on humans, irrespective of strategic considerations. Conclusions based on such a comparison are therefore problematic.

It is worth noting that our sample only included male subjects because we wanted to reduce within sample variance and because risk preferences are known to vary with gender. It remains therefore unclear to which extend our conclusions can be generalized.

Another limitation of our design is that although in the treatments with Human-Driven Uncertainty the distribution of outcomes was determined by human decisions, that lottery would ultimately be resolved by the computer drawing one random decision from that distribution. It thus can be argued that our Human-Driven Uncertainty treatments are only partially human-driven. Several studies show that subjects may ascribe strategic interests to the experimenter when programming the algorithm and it is thus unclear how findings with such mechanisms relate to uncertainty where there is no strategic interest (Oechssler and Roomets 2015; Dominiak and Duersch 2015). However, since the involvement of the computer was the same across treatments, we do not expect it to have qualitatively changed the results of the experiment. If we interpret strategic interaction as a lottery where the outcome depends on a second player, the results of this paper suggest that the nature of the second player 
may be an important feature in itself, irrespective of strategic considerations. On modern stock markets for example human traders are partially replaced by algorithmic traders and it remains unclear how, irrespective of any actual difference in trading, the new type of agent affects human traders and the market as a whole (as a first experiment on this see Farjam and Kirchkamp (2018)). Although many algorithmic traders can act quite autonomous on markets, it remains unclear to which extend depending on such agents is perceived as Human-Driven or Computerized Uncertainty. Furthermore, we do not claim that Computerized Uncertainty is equivalent to all other types of non-human uncertainty (e.g. the aforementioned natural or mechanistic uncertainty). In fact, depending on semi-autonomous algorithms may be seen as a gray area between many kinds of uncertainty. In a time where the number of interactions between humans and machines and algorithms grows inexorably and more and more human-human interaction is replaced by human-machine interaction it is of great importance to study exactly how humans perceive strategic interaction with machines different from strategic interaction with humans.

\section{Acknowledgement}

We thank the Max Planck Society for financial support through the International Max Planck Research School on Adapting Behavior in a Fundamentally Uncertain World. Special thanks also to Alexia Gaudeul, Oliver Kirchkamp, Anna Merkel and the participants of the 2015 IMPRS Uncertainty Summer School in Jena for their feedback and ideas on designing this experiment.

\section{References}

Abdellaoui, M., Baillon, A., Placido, L., Wakker, P. P., 2011. The rich domain of uncertainty: Source functions and their experimental implementation. American 
Economic Review 101 (2), 695-723.

Andersen, S., Harrison, G. W., Lau, M. I., Rutström, E. E., 2006. Elicitation using multiple price list formats. Experimental Economics 9 (4), 383-405.

Becker, G. M., DeGroot, M. H., Marschak, J., 1964. Measuring utility by a single-response sequential method. Behavioral Science 9 (3), 226-232.

Bohnet, I., Greig, F., Herrmann, B., Zeckhauser, R., 2008. Betrayal aversion: Evidence from brazil, china, oman, switzerland, turkey, and the united states. The American Economic Review 98 (1), 294-310.

Camerer, C., Weber, M., 1992. Recent developments in modeling preferences: Uncertainty and ambiguity. Journal of Risk and Uncertainty 5 (4), 325-370.

Carrubba, C. J., Yuen, A., Zorn, C., 2007. In defense of comparative statics: Specifying empirical tests of models of strategic interaction. Political Analysis 15 (4), 465-482.

Chaminade, T., Rosset, D., Da Fonseca, D., Nazarian, B., Lutcher, E., Cheng, G., Deruelle, C., 2012. How do we think machines think? An fMRI study of alleged competition with an artificial intelligence. Frontiers in Human Neuroscience 6 (103).

Chow, C. C., Sarin, R. K., 2001. Comparative ignorance and the ellsberg paradox. Journal of risk and Uncertainty 22 (2), 129-139.

Dana, J., Weber, R. A., Kuang, J. X., 2007. Exploiting moral wiggle room: experiments demonstrating an illusory preference for fairness. Economic Theory 33 (1), 67-80.

Dohmen, T., Falk, A., Huffman, D., Sunde, U., Schupp, J., Wagner, G. G., 2011. Individual risk attitudes: Measurement, determinants, and behavioral consequences. Journal of the European Economic Association 9 (3), 522-550.

Dominiak, A., Duersch, P., 2015. Benevolent and malevolent ellsberg games. Tech. rep., Discussion Paper Series.

Ellsberg, D., 1961. Risk, ambiguity, and the savage axioms. The Quarterly Journal of Economics 75 (4), 643-669.

Fairley, K., Sanfey, A., Vyrastekova, J., Weitzel, U., 2016. Trust and risk revisited. Journal of Economic Psychology 57, 74-85.

Farjam, M., Kirchkamp, O., 2018. Bubbles in hybrid markets: How expectations about algorithmic trading affect human trading. Journal of Economic Behavior \& 
Organization 146, 248-269.

Fischbacher, U., 2007. z-tree: Zurich toolbox for ready-made economic experiments. Experimental Economics 10 (2), 171-178.

Fox, C. R., Tversky, A., 1995. Ambiguity aversion and comparative ignorance. The quarterly journal of economics 110 (3), 585-603.

Heath, C., Tversky, A., 1991. Preference and belief: Ambiguity and competence in choice under uncertainty. Journal of Risk and Uncertainty 4 (1), 5-28.

Heinemann, F., Nagel, R., Ockenfels, P., 2009. Measuring strategic uncertainty in coordination games. The Review of Economic Studies 76 (1), 181-221.

Knight, F. H., 1921. Risk, uncertainty and profit. New York: Hart, Schaffner and Marx.

Koch, C., Penczynski, S. P., 2018. The winner's curse: Conditional reasoning and belief formation. Journal of Economic Theory 174, 57-102.

Krach, S., Hegel, F., Wrede, B., Sagerer, G., Binkofski, F., Kircher, T., 2008. Can machines think? interaction and perspective taking with robots investigated via fmri. PLoS One 3 (7), e2597.

Lauharatanahirun, N., Christopoulos, G. I., King-Casas, B., 2012. Neural computations underlying social risk sensitivity. Frontiers in Human Neuroscience 6 (213).

Li, C., Turmunkh, U., Wakker, P. P., 2018. Trust as a decision under ambiguity. Experimental Economics forthcoming.

Maffioletti, A., Santoni, M., 2005. Do trade union leaders violate subjective expected utility? some insights from experimental data. Theory and Decision 59 (3), 207-253.

McCabe, K., Houser, D., Ryan, L., Smith, V., Trouard, T., 2001. A functional imaging study of cooperation in two-person reciprocal exchange. Proceedings of the National Academy of Sciences 98 (20), 11832-11835.

McCabe, K. A., Rigdon, M. L., Smith, V. L., 2003. Positive reciprocity and intentions in trust games. Journal of Economic Behavior \& Organization 52 (2), 267-275.

Oechssler, J., Roomets, A., 2015. A test of mechanical ambiguity. Journal of Economic Behavior \& Organization 119, 153-162.

Rilling, J. K., Gutman, D. A., Zeh, T. R., Pagnoni, G., Berns, G. S., Kilts, C. D., 2002. A neural basis for social cooperation. Neuron 35 (2), 395-405. 
Rilling, J. K., Sanfey, A. G., Aronson, J. A., Nystrom, L. E., Cohen, J. D., 2004. The neural correlates of theory of mind within interpersonal interactions. Neuroimage 22 (4), 1694-1703.

Sanfey, A. G., 2007. Social decision-making: insights from game theory and neuroscience. Science 318 (5850), 598-602.

Sanfey, A. G., Rilling, J. K., Aronson, J. A., Nystrom, L. E., Cohen, J. D., 2003. The neural basis of economic decision-making in the ultimatum game. Science 300 (5626), $1755-1758$.

Sonsino, D., Benzion, U., Mador, G., 2002. The complexity effects on choice with uncertainty-experimental evidence. The Economic Journal 112 (482), 936-965.

Trautmann, S. T., Vieider, F. M., Wakker, P. P., 2011. Preference reversals for ambiguity aversion. Management Science 57 (7), 1320-1333.

Van Huyck, J. B., Battalio, R. C., Beil, R. O., 1991. Strategic uncertainty, equilibrium selection, and coordination failure in average opinion games. The Quarterly Journal of Economics 106 (3), 885-910. 


\section{Appendix A. Instructions}

This subsection contains the instructions in each treatment translated to English. To get an idea of the general length of the original instructions the German versions are printed in italics for the first four parts of the experiment.

Appendix A.1. Computerized Risk

We took 10 cards from a large stack of cards and shuffled these cards. On 5 of these cards an $A$ is written, on the other 5 cards a $B$ is written.

The computer will draw one of the 10 cards randomly. Guess whether there will be an $A$ or $B$ on the card.

Wir haben aus einem großen Stapel mit Karten 10 Karten genommen und diese zu einem Stapel gemischt. Auf 5 dieser Karten steht A, auf den anderen 5 Karten steht ein $\mathrm{B}$.

Der Computer wählt eine der 10 Karten zufällig aus. Raten Sie ob auf der ausgewählten Karte A oder B steht.

\section{Appendix A.2. Computerized Ambiguity}

We took 10 cards from a large stack of cards and shuffled these cards. On 0 to 10 cards (the exact number is unknown to you) an $A$ is written, on the other ones a $B$ is written.

The computer will draw one of the 10 cards randomly. Guess whether there will be an $A$ or $B$ on the card.

Wir haben aus einem großen Stapel mit Karten 10 Karten genommen und diese zu einem Stapel gemischt. Auf O bis 10 Karten (die genaue Zahl wissen Sie nicht) steht $\mathrm{A}$, auf den anderen $\mathrm{B}$.

Der Computer wählt eine der 10 Karten zufällig aus. Raten Sie ob auf der ausgewählten Karte A oder B steht. 
Appendix A.3. Human-Driven Risk

We conducted a survey on the campus among 10 people and showed them two different pictures. 5 of the participants preferred picture $A$, the other 5 participants preferred picture $B$.

The computer will draw one random participant from the 10. Guess whether the participant drawn preferred picture $A$ or $B$.

Wir haben in einer Befragung auf dem Campus 10 Menschen zwei verschiedene Bilder gezeigt. 5 der Befragten bevorzugten Bild A, die anderen 5 Befragten bevorzugten Bild $\mathrm{B}$.

Der Computer wählt einen der 10 Befragten zufällig aus. Raten Sie ob der ausgewählte Befragte Bild A oder B bevorzugte.

Appendix A.4. Human-Driven Ambiguity

We conducted a survey on the campus among 10 people and showed them two different pictures. 0 to 10 participants (the exact number is unknown to you) of the participants preferred picture $A$, the others preferred picture $B$.

The computer will draw one random participant from the 10. Guess whether the participant drawn preferred picture $A$ or $B$.

Wir haben in einer Befragung auf dem Campus 10 Menschen zwei verschiedene Bilder gezeigt. 0 bis 10 Befragte (die genaue Zahl wissen Sie nicht) bevorzugten Bild $\mathrm{A}$, die anderen Bild $\mathrm{B}$.

Der Computer wählt einen der 10 Befragten zufällig aus. Raten Sie ob der ausgewählte Befragte Bild A oder B bevorzugte.

Appendix A.5. Choice Task 5

In the following table (Table A.1) 2 of the 4 parts of the experiment are put next to each other. Indicate per row which of the parts of the experiment you prefer. The 
computer will randomly chose one of the rows. The part of the experiment that you preferred in the determined row will be played as an additional part of this experiment. 6

\begin{tabular}{|c|c|}
\hline Option A & Option B \\
\hline \hline 1st part of the experiment & 2nd part of the experiment \\
\hline 1st part of the experiment & 3rd part of the experiment \\
\hline 1st part of the experiment & 4th part of the experiment \\
\hline 2nd part of the experiment & 3rd part of the experiment \\
\hline 2nd part of the experiment & 4th part of the experiment \\
\hline 3rd part of the experiment & 4th part of the experiment \\
\hline
\end{tabular}

Table A.1: Table used for pairwise comparisons of tasks

\section{Appendix B. Becker-DeGroot-Marschak elicitation mechanism}

In a pilot experiment the Becker-DeGroot-Marschak (BDM) mechanism was used (Becker et al. 1964) to elicit subjects' $(\mathrm{N}=71)$ preferences with regard to the treatments. Figure B.1 compares the results obtained with the help of the BDM mechanism with those from the experiment discussed in the section 3 . The distributions of preferences hardly differ between those two elicitation mechanisms.

\footnotetext{
${ }^{6}$ Additionally subjects got a description of each part of the experiment as shown in the previous subsections.
} 

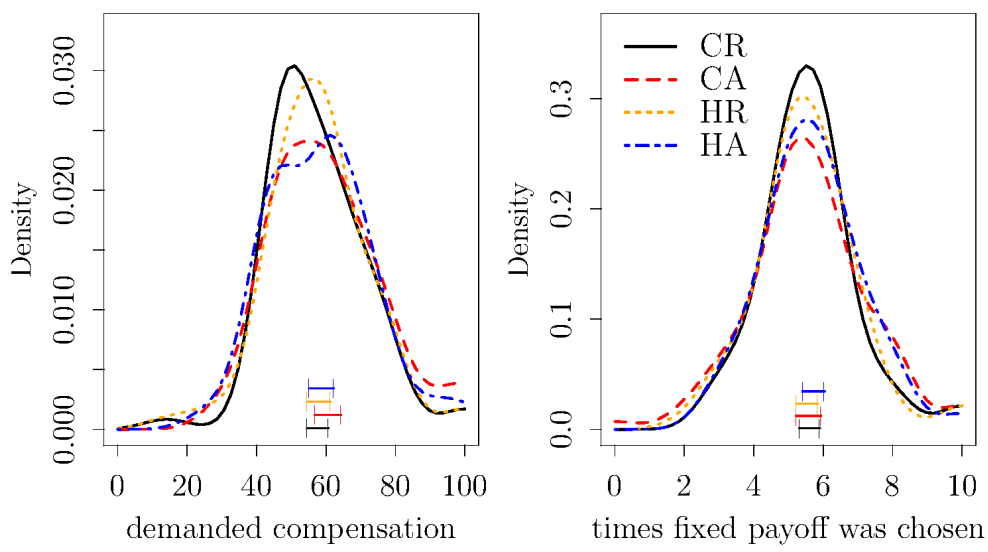

Figure B.1: Left, preferences w.r.t. the treatments elicited by the BDM-mechanism; Right, elicited with the help of a multiple price list. Per treatment the $95 \%$ bootstrap confidence interval of the mean is shown.

\section{Appendix C. Multiple Price List}

Table C.1 shows the multiple price list used to elicit subjects preferences regarding the lottery played in each treatment. In every subjects can choose between a sure payoff (Option A) and participating in the lottery (Option B). At the end of the experiment one of the rows was selected randomly for payoff according to the decision in that row. 


\begin{tabular}{|c|c|}
\hline Option A & Option B \\
\hline \hline Get 9 ECU for sure & your payoff depends on the lottery \\
\hline Get 19 ECU for sure & your payoff depends on the lottery \\
\hline$\ldots$ & $\cdots$ \\
\hline Get 89 ECU for sure & your payoff depends on the lottery \\
\hline Get 99 ECU for sure & your payoff depends on the lottery \\
\hline
\end{tabular}

Table C.1: The multiple price list presented to subjects after each treatment 